\title{
Sopra \\ le funzioni di una variabile complessa
}

\author{
(del prof. Ulisse Dini, a Pisa.)
}

C

Uauchy ha dato lo sviluppo in serie di una funzione di una variabile complessa, monodroma finita e continua essa e le sue derivate prime dentro un cerchio, in funzione dei valori che essa ha sulla circonferenza. I signori Laurent e Neumann hanno poi generalizzato il teorema di $\mathrm{C}_{\text {aUchy, }}$ dando gli sviluppi corrispondenti di una funzione per gli spazii compresi fra due cerchi concentrici o fra due ellissi omofocali, in funzione sempre dei valori che essa ha sul contorno di questi spazii. Ma una funzione di una variabile complessa è pienamente determinata in un dato campo, quando sono dati i valori della sua parte reale sul contorno ed è dato il valore della parte immaginaria in un punto (*); quindi gli sviluppi citati contengono qualche cosa di più di quello che è strettamente necessario alla determinazione della furızione negli spazii corrispondenti; e mentre ci dicono che se esiste una funzione che ha quei dati valori sul contorno di quelli spazii, e nell'interno si mantiene monodroma finita e continua insieme alle sue derivate prime, essa puo svilupparsi in serie con quelle formole, non ci danno però il mezzo di determinare la funzione quando sono date le sole quantità che bastano effettivamente a determinarla. Per questo, credo utile di pubblicare la presente memoria nella quale do gli sviluppi di una funzione di una variabile complessa nello spazio compreso fra due cerchi concentrici e nello spazio interno o esterno ad un cerchio e fra due ellissi omofocali, in funzione dei valori della sua parte reale sul contorno e del valore della parte immaginaria in un punto.

(*) Alla dimostrazione data da Riemann della esistenza di una funzione la cui parte reale prende valori dati sul contorno, ecc., si fanno varie obbiezioni; io perd in questo lavoro ammetterò l'esistenza della funzione parte reale, colla condizione che le sue derivate prime siano finite anche sul contorno, e allora essa sara appunto quella che qui viene trovata. 
1. Sia $w=u+i v$ la funzione di una variabile complessa cercata. Sono dati i valori della parte reale $u$ sul contorno del nostro campo $G$ che noi supporremo dapprima essere quello compreso fra due cerchi concentrici, ed è dato il valore della parte immaginaria $v$ in un punto di $C$.

Si tratta di determinare $w 0 u$ e $v$ in un punto qualunque $M^{\prime}\left(x^{\prime}, y^{\prime}\right)$ pure di $C$.

Incomincieremo dal determinare $u$, e per questo ricorderemo che indicando con $r$ la distanza dal punto $M^{\prime}$ a un altro punto $M(x, y)$ in un campo $C$, qualunque esso sia, e indicando con $p$ la normale al contorno contata verso l'interno di $G$, per i teoremi di Riemann e di Green, se esisterà, come supponiamo, una funzione $u$ che nell'interno di $G$ è finita continua $e$ ad un sol valore essa e le sue derivate prime, soddisfa alla equazione $\frac{d^{2} u}{d x^{2}}+\frac{d^{2} u}{d y^{2}}=0$, e sul contorno essa prende $i$ valori dati e le sue derivate prime sono finite, questa funzione sarà unica, e il suo valore nel punto $M^{\prime}$ di $C$ sarà dato dalla formola

$$
u^{\prime}=\frac{1}{2 \pi} \int\left(\log r \frac{d u}{d p}-u \frac{d \log r}{d p}\right) d s
$$

ove l'integrale del secondo menbro es esteso a tutto il contorno di $C$.

Nel caso nostro adunque, indicando con $s$ il cerchio maggiore e con $s^{\prime}$ il cerchio minore, si avrà

$$
u^{\prime}=\frac{1}{2 \pi} \int_{s}\left(\log r \frac{d u}{d p}-u \frac{d \log r}{d p}\right) d s+\int_{s^{\prime}}\left(\log r \frac{d u}{d p}-u \frac{d \log r}{d p}\right) d s^{\prime}
$$

gli integrali essendo estesi ai cerchi $s$ e $s^{\prime}$ respettivamente; e ora per trovare $u^{\prime}$ basterà fare sparire da questa formola i valori di $\frac{d u}{d p}$ sul contorno che non sono dati.

Poniamo percio l'origine delle coordinate rettilinee e polari nel centro dei due cerchi $\theta$ indichiamo con $R$ e $R^{\prime}$ i raggi di questi cerchi $s$ es $s^{\prime}$, e con $(\rho, \theta),\left(\rho^{\prime}, \theta^{\prime}\right)$ le coordinate polari di $M$ e $M^{\prime}$. Osservando che

$$
r=\left\{\rho^{2}+\rho^{\prime 2}-2 \rho \rho^{\prime} \cos \left(\theta-\theta^{\prime}\right)\right\}^{\frac{1}{2}}=\left(\rho-\rho^{\prime} e^{i\left(\theta-\theta^{\prime}\right)}\right)^{\frac{1}{2}}\left(\rho-\rho^{\prime} e^{-i\left(\theta-\theta^{\prime}\right)}\right)^{\frac{1}{2}},
$$

si trova che fuori del cerchio di raggio $\rho^{\prime}$ è:

$$
\log r=\log \rho-\sum_{1}^{\infty} \frac{\rho^{\prime n}}{n \rho^{n}} \cos n\left(\theta-\theta^{\prime}\right)
$$


e dentro a questo cerchio:

$$
\log r_{1}=\log \rho^{\prime}-\sum_{1}^{\infty} \frac{\rho^{n}}{n \rho^{\prime n}} \cos n\left(\theta-\theta^{\prime}\right)
$$

quindi sarà su $s$ :

$$
\begin{gathered}
\log r_{s}=\log R-\sum_{1}^{\infty} \frac{\rho^{\prime n}}{n R^{n}} \cos n\left(\theta-\theta^{\prime}\right), \\
\frac{d \log r_{s}}{d \rho}=-\left(\frac{d \log r}{d p}\right)_{\rho=R}=-\frac{1}{R}-\sum_{1}^{\infty} \frac{\rho^{\prime n}}{R^{n+1}} \cos n\left(\theta-\theta^{\prime}\right),
\end{gathered}
$$

e su $s^{\prime}$ :

$$
\begin{gathered}
\log r_{s^{\prime}}=\log \rho^{\prime}-\sum_{1}^{\infty} \frac{R^{\prime n}}{n \rho^{\prime n}} \cos n\left(\theta-\theta^{\prime}\right), \\
\frac{d \log r_{s^{\prime}}}{d p}=\left(\frac{d \log r_{1}}{d \rho}\right)_{\rho=R^{\prime}}=-\sum_{1}^{\infty} \frac{R^{\prime \prime t-1}}{\rho^{\prime n}} \cos n\left(\theta-\theta^{\prime}\right) ;
\end{gathered}
$$

e siccome alle serie che qui compariscono può applicarsi l'integrazione rispetto ad $s$ e a $s^{\prime}$, si avrà dalla (2):

$$
\left.\begin{array}{c}
u^{\prime}=\frac{1}{2 \pi}\left(\int_{s} \frac{u}{R} d s+\int_{s} \log R \frac{d u}{d p} d s+\int_{s^{\prime}} \log \rho^{\prime} \frac{d u}{d p} d s^{\prime}\right) \\
+\frac{1}{2 \pi} \sum_{1}^{\infty}\left\{\int_{s} \frac{\rho^{\prime n}}{R^{n+1}} \cos n\left(\theta-\theta^{\prime}\right) d s+\int_{s^{\prime}} u \frac{R^{\prime n-1}}{\rho^{\prime n}} \cos n\left(\theta-\theta^{\prime}\right) d s^{\prime}-\right. \\
\left.\frac{1}{n} \int_{s} \frac{\rho^{\prime n}}{R^{n}} \cos n\left(\theta-\theta^{\prime}\right) \frac{d u}{d p} d s-\frac{1}{n} \int_{s^{\prime}} \frac{R^{\prime n}}{\rho^{\prime n}} \cos n\left(\theta-\theta^{\prime}\right) \frac{d u}{d p} d s^{\prime}\right\} ;
\end{array}\right\}
$$

e quindi basterà ora fare sparire $\frac{d u}{d p}$ dalle due somme

$$
\begin{gathered}
\int_{s} \log R \frac{d u}{d p} d s+\int_{s} \log \rho^{\prime} \frac{d u}{d p} d s^{\prime}, \\
\int_{s} \frac{\rho^{\prime n}}{R^{n}} \cos n\left(\theta-\theta^{\prime}\right) \frac{d u}{d p} d s+\int_{s^{\prime}} \frac{R^{\prime n}}{\rho^{\prime n}} \cos n\left(\theta-\theta^{\prime}\right) \frac{d u}{d p} d s^{\prime} .
\end{gathered}
$$

Per questo (seguendo un processo suggeritomi dal prof. Betri, e giả da lui applicato nella sua memoria Sopra le funzioni sferiche) userò del principio che, se $F$ è una funzione di $x$ e $y$ che nell'interno di $G$ e sul conAnnali di Matematica, tomo IV. 
torno si mantiene finita continua e a un sol valore, essa e le sue derivate prime, e soddisfa alla equazione $\frac{d^{2} F}{d x^{2}}+\frac{d^{2} F}{d y^{2}}=0$, si ha

$$
\int F \frac{d u}{d p} d s=\int u \frac{d F}{d p} d s
$$

gli integrali essendo estesi a tutto il contorno di $C$; e prendero una volta:

e un'altra:

$$
F=A \log \rho+B
$$

$$
F=M \rho^{n} \cos n\left(\theta-\theta^{\prime}\right)+\frac{N}{\rho^{n}} \cos n\left(\theta-\theta^{\prime}\right),
$$

essendo $A, B, M, N$ costanti da determinarsi.

Si trova così servendosi del primo valore di $F$ :

$$
\int_{s}(A \log R+B) \frac{d u}{d p} d s+\int_{s^{\prime}}\left(A \log R^{\prime}+B\right) \frac{d u}{d p} d s^{\prime}=-\int_{s} \frac{A}{R} u d s+\int_{\xi^{\prime}} \frac{A}{R^{\prime}} u d s^{\prime} ;
$$

e quindi prendendo $A$ e $B$ in modo che si abbia cioe prendendo:

$$
A \log R+B=\log R, \quad A \log R^{\prime}+B=\log \rho^{\prime},
$$

si avrà

$$
A=\frac{\log \frac{R}{\rho^{\prime}}}{\log \frac{R}{R^{\prime}}}, \quad B=\log R \frac{\log \frac{\rho^{\prime}}{R}}{\log \frac{R}{R^{\prime}}}
$$

$$
\int_{s} \log R \frac{d u}{d p} d s+\int_{s^{\prime}} \log \rho^{\prime} \frac{d u}{d p} d s^{\prime}=\frac{1}{\log \frac{R}{R^{\prime}}}\left(\frac{1}{R^{\prime}} \log \frac{R}{\rho^{\prime}} \int_{s^{\prime}} u d s^{\prime}-\frac{1}{R} \log \frac{R}{\rho^{\prime}} \int_{s} u d s\right)
$$

e così è calcolata la prima delle somme (4).

Prendendo invece il secondo valore di $F$ si trova:

$$
\begin{aligned}
& \int_{s}\left(M R^{n}+\frac{N}{R^{n}}\right) \cos n\left(\theta-\theta^{\prime}\right) \frac{d u}{d p} d s+\int_{s^{\prime}}\left(M R^{\prime n}+\frac{N}{R^{\prime n}}\right) \cos n\left(\theta-\theta^{\prime}\right) \frac{d u}{d p} d s= \\
& =n \int_{s} u\left(M R^{\prime n-1}-\frac{N}{R^{\prime n+1}}\right) \cos n\left(\theta-\theta^{\prime}\right) d s^{\prime}-n \int_{s} u\left(M R^{n-1}-\frac{N}{R^{n+1}}\right) \cos n\left(\theta-\theta^{\prime}\right) d s
\end{aligned}
$$

e determinando $M$ e $N$ in modo che si abbia

$$
M R^{n}+\frac{N}{R^{n}}=\frac{\rho^{\prime n}}{R^{n}}, \quad M R^{\prime n}+\frac{N}{R^{\prime n}}=\frac{R^{\prime n}}{\rho^{\prime n}},
$$


cioè prendendo:

$$
M=\frac{\rho^{\prime 2 n}-R^{\prime 2 n}}{\rho^{\prime n}\left(R^{2 n}-R^{\prime 2 n}\right)}, \quad N=\frac{R^{\prime 2 n}}{\rho^{\prime n}} \frac{R^{2 n}-\rho^{\prime 2 n}}{R^{2 n}-R^{\prime 2 n}},
$$

resterà determinata anche la seconda delle somme (4).

Sostituendo ora nella (3) per le somme (4) i valori trovati, si otterrà subito pel valore di $u^{\prime}$ :

$$
\begin{gathered}
u u^{\prime}=\frac{1}{2 \pi \log \frac{R}{R^{\prime}}}\left(\frac{1}{R^{\prime}} \log \frac{R}{\rho^{\prime}} \int_{s^{\prime}} u d s^{\prime}-\frac{1}{R} \log \frac{R^{\prime}}{\rho^{\prime}} \int_{s} u d s\right)+ \\
+\frac{1}{\pi} \sum_{1}^{\infty} \frac{R^{n-1}}{\rho^{\prime n}} \frac{\rho^{\prime 2 n}-R^{\prime 2 n}}{R^{2 n}-R^{\prime 2 n}} \int_{s} u \cos n\left(\theta-\theta^{\prime}\right) d s+ \\
\quad+\frac{1}{\pi} \sum_{1}^{\infty} \frac{R^{\prime n-1}}{\rho^{\prime n}} \frac{R^{2 n}-\rho^{\prime 2 n}}{R^{2 n}-R^{\prime 2 n}} \int_{s^{\prime}} u \cos n\left(\theta-\theta^{\prime}\right) d s^{\prime},
\end{gathered}
$$

ovvero, indicando con $u_{s}$ e $u_{s^{\prime}}$ i valori dati di $u$ su $s$ e su $s^{\prime}$,

$$
\begin{aligned}
& u^{\prime}=\frac{1}{2 \pi \log \frac{R}{R^{\prime}}}\left(\log R \int_{0}^{2 \pi} u_{s^{\prime}} d \theta-\log R^{\prime} \int_{0}^{2 \pi} u_{s} d \theta\right)+\frac{\log \rho^{\prime}}{2 \pi \log \frac{R}{R^{\prime}}} \int_{0}^{2 \pi}\left(u_{s}-u_{s^{\prime}}\right) d \theta+ \\
& +\frac{1}{\pi} \sum_{1}^{\infty} \frac{R^{n}}{\rho^{\prime n}} \frac{\rho^{\prime 2 n}-R^{\prime 2 n}}{R^{2 n}-R^{\prime 2 n}} \int_{0}^{2 \pi} u_{s} \cos n\left(\theta-\theta^{\prime}\right) d \theta+ \\
& \quad+\frac{1}{\pi} \sum_{1}^{\infty} \frac{R^{\prime n}}{\rho^{\prime n}} \frac{R^{2 n}-\rho^{\prime 2 n}}{R^{2 n}-R^{\prime 2 n}} \int_{0}^{2 \pi} u_{s^{\prime}} \cos n\left(\theta-\theta^{\prime}\right) d \theta,
\end{aligned}
$$

ovvero infine

$$
\left.\begin{array}{r}
u^{\prime}=\frac{1}{2 \pi \log \frac{R}{R^{\prime}}}\left(\log R \int_{0}^{2 \pi} u_{s^{\prime}} d \theta-\log R^{\prime} \int_{0}^{2 \pi} u_{s} d \theta\right)+\frac{\log \rho^{\prime}}{2 \pi \log \frac{R}{R^{\prime}}} \int_{0}^{2 \pi}\left(u_{s}-u_{s}^{\prime}\right) d \theta+ \\
+\frac{1}{\pi} \sum_{1}^{\infty} \frac{\rho^{\prime n}}{R^{2 n}-R^{\prime 2 n}} \int^{2 \pi}\left(R^{n} u_{s}-R^{\prime n} u_{s^{\prime}}\right) \cos n\left(\theta-\theta^{\prime}\right) d \theta+ \\
\quad+\frac{1}{\pi} \sum_{1}^{\infty} \frac{R^{n} R^{\prime n}}{\rho^{\prime n}\left(R^{2 n}-R^{\prime 2 n}\right)} \int_{0}^{2 \pi}\left(R^{n} u_{s^{\prime}}-R^{\prime n} u_{s}\right) \cos n\left(\theta-\theta^{\prime}\right) d \theta
\end{array}\right\}
$$

Tali sono le espressioni che la formola (2) ci somministra pel valore di $u^{t}$ nell'interno e anche sul contorno di $G$, quando $i$ valori di $u$ al contorno che vi compariscono sono tali che si possono soddisfare tutte le condizioni 
poste in principio. - In un altro lavoro che pubblicherò quanto prima, tornero sopra questi risultati, e allora mi occupero anche del caso in cui la funzione deve soddisfare alle stesse condizioni nell'interno del campo che si considera, e sul contorno deve soddisfare soltanto alla condizione di prendere $i$ valori dati senza che in essa avvenga rottura di continuità quando dall'interno del campo ci si avvicina indefinitamente al contorno stesso.

Osservazione. È da notare che per la formola ('7) si puo dire che se una funzione $u$ nello spazio compreso fra due cerchi concentrici è finita continua e a un sol valore, essa e le sue derivate prime, e soddisfa alla equazione $\frac{d^{2} u}{d x^{2}}+\frac{d^{2} u}{d x^{2}}=0$, e prende valori costanti sui due cerchi e su questi cerchi le sue derivate prime sono finite, essa è della forma $A \log \rho+B$, ove $A$ e $B$ sono costanti; e se i valori costanti che prende sui due cerchi sono uguali, essa è costante per tutto.

2. Passiamo ora a calcolare la funzione $v^{\prime}$.

Per questo osserviamo che si ha

e quindi sarà

$$
\frac{d v^{\prime}}{d \rho^{\prime}}=-\frac{d u^{\prime}}{\rho^{\prime} d \theta}, \quad \frac{d v^{\prime}}{d \theta^{\prime}}=\rho^{\prime} \frac{d^{\prime} u^{\prime}}{d \rho^{\prime}},
$$

$$
v^{\prime}=\int\left(-\frac{d u^{\prime}}{\rho^{\prime} d \theta^{\prime}} d \rho^{\prime}+\rho^{\prime} \frac{d u^{\prime}}{d \rho^{\prime}} d \theta^{\prime}\right)
$$

estendendo l'integrale a una curva qualunque che va dal punto $A\left(\rho_{0}, \theta_{0}\right)$ in cui è dato il valore di $v$ al punto $M^{\prime}\left(\rho^{\prime}, \theta^{\prime}\right)$.

Prendendo dunque per linea d'integrazione la linea composta della porzione di raggio vettore $\theta_{0}$ da $\rho^{\prime}=\rho_{0}$ fino a $\rho^{\prime}=\rho^{\prime}$, e della porzione di circonferenza $\rho^{\prime} \mathrm{da} \theta^{\prime}=\theta_{0}$ fino a $\theta^{\prime}=\theta^{\prime}$, e osservando che dentro lo spazio $C$ le serie $(6)$ e (7) sono convergenti indipendentemente dall'ordine dei termini, si concluderà che per tutti i punti di $G$ ad eccezione di quelli del contorno (pei quali qui resta dubbio) si ha

$$
\left.\begin{array}{rl}
v^{\prime}=\frac{\theta^{\prime}}{2 \pi \log \frac{R}{R^{\prime}}} \int_{0}^{2 \pi}\left(u_{\theta}-u_{s^{\prime}}\right) d \theta-\frac{1}{\pi} \sum_{1}^{\infty} \frac{R^{n}}{\rho^{\prime n}} \frac{\rho^{\prime 2 n}+R^{\prime 2 n}}{R^{2 n}-R^{i 2 n}} \int_{0}^{2 \pi} u_{s} \operatorname{sen} n\left(\theta-\theta^{\prime}\right) d \theta+ \\
+\frac{1}{\pi} \sum_{i}^{\infty} \frac{R^{\prime n}}{\rho^{\prime n}} \frac{\rho^{\prime 2 n}+R^{2 n}}{R^{2 n}-R^{2 n}} \int_{0}^{2 \pi} u_{\theta^{\prime}} \operatorname{sen} n\left(\theta-\theta^{\prime}\right) d \theta+c,
\end{array}\right\}
$$


Dini: Sopra le funzioni di una variabile complessa.

ovvero:

$$
\left.\begin{array}{r}
\left.v^{\prime}=\frac{\theta^{\prime}}{2 \pi \log \frac{R}{R^{\prime}}} \int_{0}^{2 \pi}\left(u_{s}-u_{s^{\prime}}\right) d \theta-\frac{1}{\pi} \int_{1}^{\infty} \frac{\rho^{\prime n}}{R^{2 n}-R^{\prime 2 n}} \int_{0}^{2 \pi}\left(R^{n} u_{s}-R^{\prime n} u_{s^{\prime}}\right) \operatorname{sen}\left(\theta-\theta^{\prime}\right) d \theta\right) \\
+\frac{1}{\pi} \sum_{1}^{\infty} \frac{R^{n} R^{\prime n}}{\rho^{\prime n}\left(R^{2 n}-R^{\prime 2 n}\right)} \int_{0}^{2 \pi}\left(R^{n} u_{s^{\prime}}-R^{\prime n} u_{s}\right) \operatorname{sen} n\left(\theta-\theta^{\prime}\right) d \theta+c,
\end{array}\right\}
$$

essendo $c$ una costante che dipende dal valore di $v$ nel punto $\left(\rho_{0}, \theta_{0}\right)$.

Cosi è trovato anche il valore di $v^{\prime}$, cioè del coefficiente di $i$ nella funzione $u+i v$, nel campo $G$, in funzione dei valori che la parte reale $u$ ha sul contorno; però, siccome supponiamo che $R^{\prime}$ non sia zero e $R$ non sia infinito, questo valore di $v^{\prime}$, contenendo il termine

$$
\frac{\theta^{\prime}}{2 \pi \log \frac{R}{R^{\prime}}} \int_{0}^{2 \pi}\left(u_{s}-u_{s^{\prime}}\right) d \theta
$$

viene finito e continuo esso e le sue derivate nell'interno dei due cerchi e soddisfa alla equazione $\frac{d^{2} v^{\prime}}{d x^{\prime 2}}+\frac{d^{2} v^{\prime}}{d y^{2}}=0$, ma, a meno che non si riduca semplicemente connesso il nostro campo con una sezione trasversa, esso non es a un sol valore altro che quando $i$ valori dati di $u$ sui due cerchi sono tali che si ha

$$
\int_{0}^{2 \pi}\left(u_{s}-u_{s^{\prime}}\right) d \theta=0 .
$$

Tutte le derivate di $v^{\prime}$ però fra $\mathrm{i}$ due cerchi oltre esser finite e continue sono anche a un sol valore.

3. Moltiplicando per $i$ questo valore (9) di $v^{\prime}$, e sommandolo col valore (7) di $u^{\prime}$, si ottiene

$$
\begin{aligned}
w^{\prime}=u^{\prime}+i v^{\prime}=\frac{1}{2 \pi \log \frac{R}{R^{\prime}}}\left(\log R \int_{0}^{2 \pi} u_{s^{\prime}} d \theta-\log R^{\prime} \int_{0}^{2 \pi} u_{s} d \theta\right)+ & \\
& +\frac{1}{2 \pi \log \frac{R}{R^{\prime}}}\left(\log \rho^{\prime}+i \theta^{\prime}\right) \int_{\theta}^{2 \pi}\left(u_{s}-u_{s^{\prime}}\right) d \theta+ \\
& +\frac{1}{\pi} \sum_{1}^{\infty} \frac{\rho^{\prime n}}{R^{2 n}-R^{2 n}} \int_{0}^{2 \pi}\left(R^{n} u_{s}-R^{\prime n} u_{s^{\prime}}\right) e^{-i n\left(\theta-\theta^{\prime}\right)} d \theta+ \\
& +\frac{1}{\pi} \sum_{1}^{\infty} \frac{R^{n} R^{\prime n}}{\rho^{i n}\left(R^{2 n}-R^{\prime 2 n}\right)} \int_{\theta}^{2 \pi}\left(R^{n} u_{s^{\prime}}-R^{\prime n} u_{s}\right) e^{i n\left(\theta-\theta^{\prime}\right)} d \theta+i c,
\end{aligned}
$$




$$
\begin{aligned}
& \text { ovvero, ponendo } \rho^{\prime} e^{i \theta^{\prime}}=z^{\prime}, \\
& \begin{aligned}
& w^{\prime}=\frac{1}{2 \pi \log \frac{R}{R^{\prime}}}\left(\log R \int_{0}^{2 \pi} u_{s^{\prime}} d \theta-\log R^{\prime} \int_{0}^{12 \pi} u_{s} d \theta+\frac{1}{2 \pi \log \frac{R}{R^{\prime}}} \log z^{\prime} \int_{\theta}^{2 \pi}\left(u_{s}-u_{s^{\prime}}\right) d \theta+\right. \\
&+\frac{1}{\pi} \sum_{1}^{\infty} \frac{z^{\prime n}}{R^{2 n}-R^{i 2 n}} \int_{0}^{2 \pi}\left(R^{n} u_{s}-R^{\prime n} u_{s^{\prime}}\right) e^{-i n \theta} d \theta+ \\
&+\frac{1}{\pi} \sum_{1}^{\infty} \frac{R^{n} R^{\prime n}}{z^{\prime n}\left(R^{2 n}-R^{(2 n}\right)} \int_{0}^{2 \pi}\left(R^{n} u_{s^{\prime}}-R^{\prime n} u_{s}\right) e^{i n \theta} d \theta+i c,
\end{aligned}
\end{aligned}
$$

e così abbiamo il valore di $w^{\prime}$ in funzione dei valori di $u$ sul contorno.

Questo valore di $w^{\prime}$ viene dato per una serie ordinata per le potenze intere positive e negative di $z^{\prime}$ e contiene inoltre un termine in $\log z^{\prime}$; quindi, non intendendo ridotto il campo $C$ semplicemente connesso, si puó dire che quando si prenderanno arbitrariamente $i$ valori della parte reale $u$ sul contorno, senza altre condizioni che quelle poste al $\$ 1$, la funzione $w$ in generale non verra monodroma, ma conterrà il termine

$$
\frac{\log z^{\prime}}{2 \pi \log \frac{R}{R^{\prime}}} \int_{0}^{2 \pi}\left(u_{s}-u_{s^{\prime}}\right) d \theta \text {; }
$$

e quindi per ogni giro attorno al centro dei due cerchi, tornando allo stesso punto, la funzione verrà aumentata della costante immaginaria

$$
\frac{i}{\log \frac{R}{R^{\prime}}} \int_{0}^{2 \pi}\left(u_{s}-u_{s^{\prime}}\right) d \theta .
$$

Questo si presenta qui come caso particolare di una proprietá generale enunciata da Riemann nella sua dissertazione inaugurale.

Le derivate di $w^{\prime}$ però fra $\mathrm{i}$ due cerchi oltre esser finite e continue saranno anche monodrome.

4. La condizione necessaria e sufficiente affinchè nello spazio compreso fra due cerchi concentrici la funzione $w$, che corrisponde ai valori $u_{s}, u_{s^{t}}$ della parte reale sul contorno, sia monodroma, viene dunque espressa dalla equazione

$$
\int_{0}^{2 \pi}\left(u_{s}-u_{s^{\prime}}\right) d \theta=0
$$


e quando questa sia soddisfatta, sarà:

$$
\left.\begin{array}{rl}
w^{\prime}=\frac{1}{2 \pi} \int_{0}^{2 \pi} u_{s} d \theta & +\frac{1}{\pi} \sum_{1}^{\infty} \frac{z^{\prime n}}{R^{2 n}-R^{i n}} \int_{0}^{2 \pi}\left(R^{n} u_{s}-R^{\prime n} u_{s^{\prime}}\right) e^{-i n \theta} d \theta+ \\
& +\frac{1}{\pi} \sum_{1}^{\infty} \frac{R^{n} R^{\prime n}}{z^{\prime n}\left(R^{2 n}-R^{\prime 2 n}\right)} \int_{0}^{2 \pi}\left(R^{n} u_{s^{\prime}}-R^{\prime n} u_{s}\right) e^{i n \theta} d \theta+i c .
\end{array}\right\}
$$

In particolare, se $u_{s^{\prime}}=u_{s}$, la funzione $w$ risulterà monodroma, e si avrà

$$
\left.\begin{array}{rl}
w^{\prime}=\frac{1}{2 \pi} \int_{0}^{2 \pi} u_{s} d \theta & +\frac{1}{\pi} \sum_{1}^{\infty} \frac{z^{\prime n}}{R^{n}+R^{n}} \int_{0}^{2 \pi} u_{s} e^{-i n \theta} d \theta+ \\
& +\frac{1}{\pi} \sum_{1}^{\infty} \frac{1}{z^{\prime n}\left(R^{-n}+R^{\prime-n}\right)} \int_{0}^{2 \pi} u_{s} e^{i n \theta} d \theta+i c .
\end{array}\right\}
$$

In generale poi si può notare che, siccome $u_{s}-u_{\theta^{\prime}}$ una funzione di $\theta$ fra 0 e $2 \pi$ che puó svolgersi in serie colla formola di Fourier, la condizione di monodromia della funzione $w$ porterà che nello sviluppo di $u_{s}-u_{s^{\prime}}$, secondo la serie di Fourter, si abbia

$$
\left.\begin{array}{rl}
u_{s}-u_{s}^{\prime} & =a_{1} \cos \theta+a_{2} \cos 2 \theta+a_{3} \cos 3 \theta+\cdots \\
& +b_{1} \operatorname{sen} \theta+b_{2} \operatorname{sen} 2 \theta+b_{3} \operatorname{sen} 3 \theta+\cdots
\end{array}\right\}
$$

cioè che manchi il termine indipendente da $\theta$; e siccome alle serie di FouRIER pud applicarsi l'integrazione (*), quando questa condizione sia soddisfatta, si avrà

$$
\begin{aligned}
w^{\prime}=\frac{1}{2 \pi} \int_{\theta}^{-\pi} u_{s} d \theta+\frac{1}{\pi} \sum_{1}^{\infty} \frac{z^{\prime n}}{R^{n}+R^{\prime n}} \int_{0}^{2 \pi} u_{s} e^{-i n \theta} d \theta+ \\
\quad+\frac{1}{\pi} \sum_{1}^{\infty} \frac{1}{z^{\prime n}\left(R^{-n}+R^{\prime}-n\right.} \int_{0}^{2 \pi} u_{s} e^{i n \theta} d \theta+ \\
\quad+\sum_{1}^{\infty} \frac{z^{\prime n} R^{\prime n}}{R^{2 n}-R^{\prime 2 n}}\left(a_{n}-i b_{n}\right)-\sum_{1}^{\infty} \frac{R^{2 n} R^{\prime n}}{z^{\prime n}\left(R^{2 n}-R^{3 n}\right)}\left(a_{n}+i b_{n}\right)+i c .
\end{aligned}
$$

5. Facciamo ora alcune osservazioni sui risultati che precedono.

(*) Queste proprieta delle serie di Fouruer sarà dimostrata nel lavoro di cui ho parlato alla fine del $\$ 1$. 
I. La condizione di monodromia (11) della funzione $w$ sarà evidentemente soddisfatta anche per due cerchi qualunque nell'interno di $C$ concentrici ai due cerchi dati $s$ e $s^{\prime}$ e vicini fra loro quanto si vuole; e quindi supponendo questi due cerchi infinitamente vicini l'uno a l'altro, si avrà evidentemente [come anche risulta subito dalle formole $(7)$ e (11)]

$$
\int_{s_{1}} \frac{d u}{d p} d s_{1}=0, \quad \mathrm{e} \int \frac{d w}{d p} d s_{1}=0,
$$

quando si estendano questi integrali a un cerchio qualunque $s_{1}$ interno a $C$ e concentrico ai due cerchi dati.

Da cio risulta che, avendo uno spazio qualunque $S$ nel quale una funzione $w$ di una variabile complessa si mantiene monodroma finita e continua essa e le sue derivate prime, e tracciando in questo spazio una curva chiusa qualunque $\sigma$ che formi il contorno completo di una porzione di $S$, o tale che si possano costruire due cerchi concentrici $s$ e $s^{\prime}$ che non taglino $\sigma$ e con ciascuno dei quali $\sigma$ formi il contorno completo di una parte di $S$, si avrà

$$
\int_{\sigma} \frac{d w}{d p} d \sigma=0
$$

giacchè nel primo caso questo risulta subito da un teorema noto, e nel secondo costruendo un terzo cerchio $s_{1}$ fra $s$ e $s^{\prime}$ e concentrico a questi, per ciỏ che precede, si avrà

$$
\int_{s_{1}} \frac{d w}{d p} d s_{1}=0
$$

e quindi, poichè per un teorema noto si ha

sarà anche

$$
\int_{s_{1}} \frac{d w}{d p} d s_{1}+\int_{\sigma} \frac{d w}{d p} d \sigma=0
$$

$$
\int \frac{d w}{d p} d \sigma=0
$$

II. Nel caso in cui la condizione di monodromia

$$
\int_{\theta}^{2 \pi}\left(u_{s}-u_{s^{\prime}}\right) d \theta=0
$$


e soddisfatta, la serie (12) che abbiamo trovata per $w^{\prime}$ si trasforma facilmente in quella di Laureat.

Indicando infatti con $w_{\varrho_{1}}$ il valore di $w$ sul cerchio di raggio $\rho_{1}$ interno a $G$ e concentrico ai cerchi $s$ e $s^{\prime}$, la (12) ci darà le formole seguenti:

$$
\begin{gathered}
\int_{0}^{2 \pi} w_{\rho_{1}} d \theta^{\prime}=-i \int_{\rho_{1}} \frac{w}{z} d z=\int_{0}^{2 \pi} u_{s} d \theta+2 \pi i c, \\
\frac{1}{\rho_{1}^{n}} \int_{0}^{2 \pi} w_{\varrho_{1}} e^{-i n \theta^{\prime}} d \theta^{\prime}=-i \int_{\rho_{1}} \frac{w}{z^{n+1}} d z=\frac{2}{R^{2 n}-R^{2 n}} \int_{0}^{2 \pi}\left(R^{n} u_{s}-R^{\prime n} u_{s^{\prime}}\right) d \theta, \\
\rho_{1}^{n} \int_{0}^{2 \pi} w_{\varrho_{1}} e^{i n \theta^{\prime}} d \theta^{\prime}=-i \int_{\rho_{1}} w z^{n-1} d z=\frac{2 R^{n} R^{\prime n}}{R^{-n}-R^{2 n}} \int_{0}^{2 \pi}\left(R^{n} u_{s^{\prime}}-R^{\prime n} u_{s}\right) d \theta,
\end{gathered}
$$

che sono abbastanza notevoli; e ora applicando queste formole una volta al cerchio di raggio $\rho$ e un'altra al cerchio di raggio $\rho_{1}$, essendo $R^{\prime}<\rho_{1}<$ $<\rho<R$, e sostituendo nella (12) si otterrá la seguente:

$$
w^{\prime}=\frac{1}{2 \pi i} \sum_{0}^{\infty} z^{\prime n} \int_{\rho} \frac{w}{z^{n+1}} d z+\frac{1}{2 \pi i} \sum_{1}^{\infty} \frac{1}{z^{\prime n}} \int_{\rho_{1}} w z^{n-1} d z
$$

che è quella di LaUrent pei due cerchi $\rho$ e $\rho_{1}$.

Da questa [come anche dalla (12) servendosi della (16)] si ottiene l'altra:

$$
w^{\prime}=\frac{1}{2 \pi i} \sum_{0}^{\infty} z^{\prime n} \int_{\rho} \frac{w}{z^{n+1}} d z+\frac{1}{2 \pi i} \sum_{1}^{\infty} \frac{1}{z^{\prime n}} \int_{\rho} w z^{n-1} d z,
$$

che fu pure data da LAURENT; e questa, contenendo soltanto i valori di $w$ sul cerchio di raggio $\rho$ interno ai due $s$ e $s^{\prime}$, ci mostra che quando esiste una funzione di una variabile complessa che è monodroma finita e continua insieme alle sue derivate nel campo compreso fra due cerchi concentrici e prende dati valori su un terzo cerchio $\rho$ interno e concentrico a questo, essa sarà data dalla serie (18).

III. Se si cercassero le funzioni $u, v$ e $w$ entro an cerchio di raggio $R$, si troverebbe coi metodi precedenti 


$$
\begin{aligned}
& u^{\prime}=\frac{1}{2 \pi} \int_{0}^{2 \pi} u_{s} d \theta+\frac{1}{\pi} \sum_{1}^{\infty} \frac{\rho^{\prime n}}{R^{n}} \int_{0}^{2 \pi} u_{s} \cos n\left(\theta-\theta^{\prime}\right) d \theta \\
& v^{\prime}=-\frac{1}{\pi} \sum_{1}^{\infty} \frac{\rho^{\prime n}}{R^{n}} \int_{0}^{2 \pi} u_{s} \operatorname{sen} n\left(\theta-\theta^{\prime}\right) d \theta+c \\
& w^{\prime}=\frac{1}{2 \pi} \int_{0}^{2 \pi} u_{s} d \theta+\frac{1}{\pi} \sum_{i}^{\infty} \frac{z^{\prime n}}{R^{n}} \int_{0}^{2 \pi} u_{s} e^{-i n \theta} d \theta+i c
\end{aligned}
$$

quindi in questo caso la funzione $w$ viene monodroma qualunque siano i valori dati per la parte reale sul contorno; e il valore della sua parte reale nel centro del cerchio è

$$
\frac{1}{2 \pi} \int_{0}^{2 \pi} u_{s} d \theta
$$

Volendo invece una funzione come $w$ di una variabile complessa $z$ nello spazio esterno al cerchio $R$, quando sono dati i valori della sua parte reale su questo cerchio, ed è posta la condizione che all'infinito la funzione sia costante e il prodotto $\frac{d w}{d z} z^{2}$ si mantenga finito, si osserverà che ponendo $z=\frac{R^{2}}{z_{1}}$, e prendendo $z_{1}$ per variabile, $w$ verrà una funzione di $z_{1}$ che sarà monodroma finita e continua essa e le sue derivate prime nel cerchio di raggio $R$ (descritto sul piano di $z_{1}$ ), e su questo cerchio la sua parte reale prenderå ancora $\mathrm{i}$ valori dati $\mathrm{ma}$ in senso opposto; e quindi le formole corrispondenti a questo caso saranno quelle che si ottengono dalle precedenti (19) cangiandovi $\rho^{\prime}$ in $\frac{R^{2}}{\rho^{\prime}}, \theta^{\prime}$ in $-\theta^{\prime}$, e $u_{s}$ in $u_{s}(-\theta)$, e si potranno ridurre alle seguenti:

$$
\begin{aligned}
& u^{\prime}=\frac{1}{2 \pi} \int_{0}^{2 \pi} u_{s} d \theta+\frac{1}{\pi} \sum_{1}^{\infty} \frac{R^{n}}{\rho^{\prime n}} \int_{0}^{2 \pi} u_{s} \cos n\left(\theta-\theta^{\prime}\right) d \theta \\
& v^{\prime}=\frac{1}{2 \pi} \sum_{1}^{\infty} \frac{R^{n}}{\rho^{\prime n}} \int_{0}^{2 \pi} u_{s} \operatorname{sen} n\left(\theta-\theta^{\prime}\right) d \theta+c \\
& w^{\prime}=\frac{1}{2 \pi} \int_{0}^{2 \pi} u_{\varepsilon} d \theta+\frac{1}{\pi} \sum_{1}^{\infty} \frac{R^{\prime \pi}}{z^{\prime n}} \int_{0}^{2 \pi} u_{s} e^{i n \theta} d \theta+i c
\end{aligned}
$$


quando vi si cangi $\theta$ in $2 \pi-\theta$ e si supponga $u_{s}(\theta-2 \pi)=u_{s}(\theta)=u_{s}$; e sotto questa forma si vede che esse, come le (19), risultano da quelle date pel caso di due cerchi $R$ e $R^{\prime}$, quando vi si faccia rispettivamente $R=\infty$ o $R^{\prime}=0$, e si cangi poi nel primo caso $R^{\prime}$ in $R$, e $u_{s^{\prime}}$ in $u_{s}$.

6. Cerchiamo ora lo sviluppo della nostra funzione $w$ di una variabile complessa nel campo racchiuso da due ellissi omofocali, in funzione dei valori che la parte reale ha sul contorno.

Per questo potremmo ancora seguire il metodo precedente; ma io preferisco di seguirne un altro (che può applicarsi anche in altri casi), deducendo uno sviluppo della funzione cercata da quello che abbiamo trovato nel caso di due cerchi.

Indichiamo percio con $z_{1}$ la nostra variabile complessa; con $2 d$ la distanza focale delle ellissi date, e con $\alpha$ e $\beta$ i parametri isometrici del doppio sistema di ellissi e iperbole omofocali di cui queste ellissi date fanno parte. Le formole che esprimono $x_{1}, y_{1}, z_{1}$ per $\alpha$ e $\beta$ saranno le seguenti

$$
x_{1}=d \cosh \alpha \cos \beta, \quad y_{1}=d \operatorname{senh} \alpha \operatorname{sen} \beta, \quad z_{1}=d \cos (\beta-i \alpha) ;
$$

e con queste, facendo variare $\alpha$ da 0 a $\infty$ e $\beta$ da 0 a $2 \pi$, si otterranno tutti $i$ punti del nostro piano $z_{1}$. Le ellissi date corrisponderanno a due valori particolari $\alpha_{0}$ e $\alpha_{1}$ di $\alpha$.

Consideriamo ora un altro piano $z$, e rappresentiamolo su $z_{1}$ in modo che si abbia la similitudine nelle parti infinitesime. Il sistema di coordinate polari $(\rho, 0)$ costituisce su questo piano un doppio sistema di coordinate ortogonali e isoterme di cui i parametri isometrici sono $\log \rho$ e $\theta$; quindi, poichè anche le linee $\alpha, \beta$ su $z_{1}$ sono esse pure ortogonali e isoterme, potremo stabilire la corrispondenza in modo da ottenere che alle circonferenze $\rho$ corrispondano le ellissi $\alpha$, e alle semi-rette $\theta$ i rami di iperbola $\beta$. Per questo basterà porre

$$
\log \rho+i \theta=m(\alpha \pm i \beta)+n+i n^{\prime}
$$

essendo $m, n, n^{\prime}$ costanti arbitrarie reali; e se vogliamo che percorrendo una intera circonferenza $\rho$ su $z$ si venga a percorrere una intiera ellisse $\alpha$ su $z_{1}$ e nello stesso senso, bisognerà prendere $m= \pm 1$ secondochè si prende il segno superiore o l'inferiore di $i \beta$. Inoltre potremo prendere $n^{\prime}=0$, poichè ciò equivale a fare corrispondere l'asse delle $x_{1}$ a quello delle $x$, e potremo porre $n=-\log c$, essendo $c$ una costante positiva.

$\mathrm{Si}$ avrà così per la nostra corrispondenza

$$
\log c+\log \rho+i \theta=\log c z= \pm \alpha+i \beta,
$$


e per la (21) questa corrispondenza potrà esprimersi anche colle formole

$$
x_{1}=d \cosh ( \pm \log c \rho) \cos \theta, \quad y_{1}=d \operatorname{senh}( \pm \log c \rho) \operatorname{sen} \theta
$$

e noi vediamo perció che quando si prenda il segno superiore di $\alpha$ nella (22), 0 il segno superiore di $\log c \rho$ in queste ultime, $\rho$ non potrà essere minore di $\frac{1}{c}$, e quando si prenda il segno inferiore $\rho$ non potrà essere maggiore di $\frac{1}{c}$; e quindi, osservando anche che per $\rho=\frac{1}{c}$ si ha sempre dalle (23) $x_{1}=d \cos \theta, y_{1}=0$, e per $\theta=0$ si ha $x_{1}=d \cosh ( \pm \log c \rho), x_{1}=0$, si potrà dire che le rappresentazioni su $z_{1}$ dei punti di $z$ esterni al cerchio di raggio $\frac{1}{c}$, e quelle dei punti interni a questo cerchio ricuoprono ambedue il piano $z_{1}$, e per tutte e due queste rappresentazioni il cerchio limite $\frac{1}{c}$ viene rappresentato sulla porzione di asse delle $x_{1}$ compreso fra $\mathrm{i}$ fuochi, mentre le altre porzioni di quest'asse rappresentano la porzione di asse delle $x$ che si trova nel campo rappresentato.

Qui prenderemo il segno superiore di $\alpha$ e $\operatorname{di} \log c \rho$, intendendo percio che sia sempre $\rho \geq \frac{1}{c}$; allora la corrispondenza fra $i$ punti dei due piani $z$ e $z_{1}$ potrà esprimersi colla equazione

$$
z_{1}=\frac{d}{2}\left(c z+\frac{1}{c z}\right)
$$

e al cerchio di raggio $\rho$ corrisponderà l'ellisse $\alpha$ per la quale $\alpha=\log c \rho, 0$ $\rho=\frac{e^{x}}{c}$, e il cui semi-asse maggiore $a$ è dato dalla formola $a=\frac{d}{2}\left(c \rho+\frac{1}{c \rho}\right)$. Le ellissi $\alpha_{0}$ e $\alpha_{1}$ corrisponderanno ai due cerchi $R$ e $R^{\prime}$ pei quali $R=\frac{e^{\alpha_{0}}}{c}$, $R^{\prime}=\frac{e^{\alpha_{1}}}{c}$, e se $\alpha_{0}>\alpha_{1}$ sarà $R>R^{\prime}$, e allo spazio racchiuso fra questi cerchi corrisponderà lo spazio fra le dette ellissi.

Inoltre per la (24) si avrà

$$
c d z=z_{1}+\sqrt{z_{1}^{2}-d^{2}}
$$

e il radicale dovrà essere preso in modo che per $z_{1}$ positivo e maggiore di $d$ sia positivo; e cosi, fra le due ellissi, $\sqrt{z_{1}^{2}-d^{2}}$ sarà bene determinato per ogni valore di $z_{1}$. 
Con ciò si vede subito che una funzione di una variabile complessa $z$, monodroma finita e continua essa e le sue derivate prime nello spazio racchiuso fra due cerchi concentrici, pud trasformarsi in una funzione di una altra variabile complessa $z_{1}$ dotata delle stesse proprietà nello spazio racchiuso fra due ellissi omofocali; e ora siccome si ha $\rho=\frac{e^{\alpha}}{c}, \theta=\beta, R=\frac{e^{\alpha_{0}}}{c}$, $R^{\prime}=\frac{e^{x_{1}}}{c}$, e $R>R^{\prime}$ se $\alpha_{0}>\alpha_{1}$, si conclude subito che, per avere i valori cercati di $u^{\prime}, v^{\prime}, w^{\prime}$ fra le due ellissi $\alpha_{0}$ e $\alpha_{1}$ in funzione dei valori di $u$ sul contorno e in coordinate ellittiche $\alpha$ e $\beta$, non vi è altro da fare che sostituire nelle formole (7) (9) e (10) per $\rho, \theta, R, R^{\prime}$ questi loro valori. Si trova cosi

$$
\begin{aligned}
& u^{\prime}=\frac{1}{2 \pi\left(x_{0}-\alpha_{1}\right)}\left(\alpha_{1} \int_{0}^{2 \pi} u_{1} d \beta-\alpha_{1} \int_{0}^{2 \pi} u_{0} d \beta\right)+\frac{\alpha^{\prime}}{2 \pi\left(\alpha_{0}-\alpha_{1}\right)} \int_{0}^{2 \pi}\left(u_{0}-u_{1}\right) d \beta+ \\
& +\frac{1}{\pi} \sum_{1}^{\infty} \frac{e^{n a^{\prime}}}{e^{2 n \alpha_{0}}-e^{z n \alpha_{1}}} \int_{0}^{2 \pi}\left(e^{n \alpha_{0}} u_{0}-e^{n \alpha_{1}} u_{1}\right) \cos n\left(\beta-\beta^{\prime}\right) d \beta+ \\
& +\frac{1}{\pi} \sum_{1}^{\infty} \frac{e^{n\left(\alpha_{0}+\alpha_{1}-\alpha^{\prime}\right)}}{e^{2 n \alpha_{0}}-e^{2 n \alpha_{1}}} \int_{0}^{2 \pi}\left(e^{n \alpha_{0}} u_{1}-e^{n \alpha_{1}} u_{0}\right) \cos n\left(\beta-\beta^{\prime}\right) d \beta \\
& v^{\prime}=\frac{\beta^{\prime}}{2 \pi\left(\alpha_{0}-\alpha_{1}\right)} \int_{0}^{2 \pi}\left(u_{0}-u_{1}\right) d \beta- \\
& -\frac{1}{\pi} \sum_{1}^{\infty} \frac{e^{n \alpha^{\prime}}}{e^{2 n \alpha_{0}}-e^{2 n \alpha_{1}}} \int_{0}^{2 \pi}\left(e^{n \alpha_{0} u_{0}}-e^{n \alpha_{1}} u_{1}\right) \operatorname{sen} n\left(\beta-\beta^{\prime}\right) d \beta+ \\
& +\frac{1}{\pi} \sum_{1}^{\infty} \frac{e^{n\left(\alpha_{0}+\alpha_{1}-\alpha^{\prime}\right)}}{e^{2 n \alpha_{0}}-e^{2 n \alpha_{1}}} \int_{0}^{2 \pi}\left(e^{n \alpha_{0}} u_{1}-e^{n \alpha_{1}} u_{0}\right) \operatorname{sen} n\left(\beta-\beta^{\prime}\right) d \beta+c \\
& w^{\prime}=\frac{1}{2 \pi\left(\alpha_{0}-\alpha_{1}\right)}\left(\alpha_{0} \int_{0}^{2 \pi} u_{1} d \beta-\alpha_{1} \int_{0}^{2 \pi} u_{0} d \beta\right)+\frac{\alpha^{\prime}+i \beta^{\prime}}{2 \pi\left(\alpha_{0}-\alpha_{1}\right)} \int_{0}^{2 \pi}\left(u_{0}-u_{1}\right) d \beta+ \\
& +\frac{1}{\pi} \sum_{1}^{\infty} \frac{e^{n\left(\alpha^{\prime}+i \beta^{\prime}\right)}}{e^{2 n \alpha_{0}}-e^{2 n \alpha_{1}}} \int_{0}^{9 \pi}\left(e^{n \alpha_{0}} u_{0}-e^{n \alpha_{1}} u_{1}\right) e^{-i n \beta} d \beta+ \\
& +\frac{1}{\pi} \sum_{1}^{\infty} \frac{e^{n\left(\alpha_{0}+\alpha_{1}-\alpha^{\prime}-i \beta^{\prime}\right)}}{e^{2 n \alpha_{0}}-e^{2 n \alpha_{1}}} \int_{0}^{2 \pi}\left(e^{n \alpha_{0}} u_{1}-e^{n \alpha_{4}} u_{0}\right) e^{i n \beta} d \beta+i c,
\end{aligned}
$$

ore $u_{0}$ e $u_{1}$ indicano $\mathrm{i}$ valori dati di $u$ sopra le ellissi $\alpha_{0}$ e $\alpha_{1}$ rispettivamente. 
174 Dini: Sopra le funzioni di una variabile complessa.

Volendo introdurre in $w^{\prime}$ la variabile $z_{1}^{\prime}$ che ora possiamo chiamare $z^{\prime}$, si vede dalle (21) che basta porre $\frac{z^{\prime}+\sqrt{z^{\prime 2}-d^{2}}}{d}$ al posto di $e^{\alpha^{\prime}+i \beta^{\prime}}$, e intendere che il radicale sia preso ancora in modo che per $z^{\prime}$ positivo e maggiore di $d$ sia positivo.

La condizione di monodromia di $w$ viene ora espressa dalla equazione

$$
\int_{0}^{2 \pi}\left(u_{0}-u_{1}\right) d \beta=0 .
$$

Supponendo nelle formole precedenti $\alpha_{1}=0$, si ottengono i valori di $u^{\prime}$, $v^{\prime}, w^{\prime}$ entro una ellisse nella quale è fatta una sezione rettilinea limitata ai due fuochi, in funzione dei valori della parte reale sull'ellisse e su questa sezione rettilinea. Il valore di $w^{\prime}$ è

$$
\begin{aligned}
& w^{\prime}=\frac{1}{2 \pi} \int_{0}^{2 \pi} u_{1} d \beta+\frac{\alpha^{\prime}+i \beta^{\prime}}{2 \pi \alpha_{0}} \int_{0}^{2 \pi}\left(u_{0}-u_{1}\right) d \beta+ \\
& +\frac{1}{\pi} \sum_{1}^{\infty} \frac{e^{n\left(\alpha^{\prime}+i \beta^{\prime}\right)}}{e^{2 n \alpha_{0}}-1} \int_{0}^{2 \pi}\left(e^{n \alpha_{0}} u_{0}-u_{1}\right) e^{-i n \beta} d \beta+ \\
& +\frac{1}{\pi} \sum_{5}^{\infty} \frac{e^{n\left(\alpha_{0}-\alpha^{\prime}-i \beta^{\prime}\right)}}{e^{2 n \alpha_{0}}-1} \int_{0}^{2 \pi}\left(e^{n \alpha_{0}} u_{1}-u_{0}\right) e^{i n \beta} d \beta+i c .
\end{aligned}
$$

Dalla formola di LAURENT poi se ne deduce una analoga pel caso attuale cambiandovi $z$ in $z+\sqrt{z^{2}-d^{2}}$ e $z^{\prime}$ in $z^{\prime}+\sqrt{z^{\prime 2}-d^{z}}$.

Pisa, 15 ottobre 1870 . 